\title{
On the Uplink MAC Performance of a Drive-Thru Internet
}

\author{
Yanyan Zhuang, Student Member, IEEE, Jianping Pan, Senior Member, IEEE, \\ Vidhoon Viswanathan, and Lin Cai, Senior Member, IEEE
}

\begin{abstract}
As IEEE 802.11 access points (APs) open up services to mobile clients, opportunistic access to the roadside communication infrastructures from traveling vehicles has become more prominent and has drawn considerable attention. In particular, data uploading from traveling vehicles has a great potential for many vehicular ad hoc network applications, where both the intermittent connectivity and the time-varying vehicle arrivals have presented significant challenges to the current analytical models. Our focus is on the carrier sense multiple access with collision avoidance-based medium access control (MAC) layer performance in a last-hop drive-thru Internet, considering both the contention nature of the uplink and the realistic vehicle traffic model. Analytical and simulation results show the intrinsic relationships among the vehicle density and speed, the coverage of the AP, the achievable throughput, and the total amount of data uploaded by vehicles. We further investigate the efficacy of an admission control scheme by the AP for achieving an optimal operating region that has both a high throughput and a large amount of data uploaded from each drive-thru vehicle.
\end{abstract}

Index Terms-IEEE 802.11 medium access control (MAC), uplink performance analysis, vehicular networks.

\section{INTRODUCTION}

$\mathbf{T}$ HE NEED FOR on the move access to the Internet has promoted the creation and adoption of the mobile Internet via Third-Generation, WiMax, etc. For a vehicle and its passengers, however, there is only limited access to the infrastructure while driving. Although cellular networks can provide the Internet access services, the high cost of a data plan subscription and the relatively low capacity have largely limited the service availability and affordability of cellular networks. With the penetration of IEEE-802.11-based networks, wireless Internet access points (APs) are widely deployed in city blocks and becoming increasingly available along highways. Their lowcost high-capacity low-coverage nature yields opportunistic access to roadside communication infrastructures from travel-

Manuscript received January 31, 2011; revised September 5, 2011 and January 4, 2012; accepted February 15, 2012. Date of publication March 6, 2012; date of current version May 9, 2012. This work was supported in part by the Natural Sciences and Engineering Research Council of Canada. The work of V. Viswanathan was also supported by Mitacs through the Globalink Internship Program during his 2010 summer internship at the University of Victoria, Victoria, BC, Canada. The review of this paper was coordinated by Prof. P. Langendoerfer.

Y. Zhuang and J. Pan are with the Department of Computer Science, University of Victoria, Victoria, BC V8W 3P6, Canada (e-mail: yyzhuang@uvic.ca; pan@uvic.ca).

V. Viswanathan is with the Texas Instruments Research and Development, Bangalore 560 093, India (e-mail: vidhoon.ceg@gmail.com).

L. Cai is with the Department of Electrical and Computer Engineering, University of Victoria, Victoria, BC V8P 5C2, Canada (e-mail: cai@ece.uvic.ca).

Digital Object Identifier 10.1109/TVT.2012.2189424 ing vehicles. With the proliferation of public WiFi initiatives, such drive-thru Internet connections have become increasingly popular and attracted considerable attention.

Various studies have demonstrated the feasibility of such drive-thru Internet. However, the existing work on drive-thru Internet has mainly studied the downlink performance (from the AP to vehicles) [5]-[7], or the uplink performance (from vehicles to the AP) without considering the contention access, e.g., due to a limited number of vehicles involved [8], [9]. In a drive-thru Internet, an important class of application is to upload data from vehicles to roadside units, with sensory data collected or scenic data captured along the way. For timesensitive safety applications, the uplink performance determines the message dissemination latency, such as uploading video clips from vehicles that have passed a crash site. Vehicles driving through the coverage region of an AP may have a high volume of data to saturate the uplink. The target of this paper is the uplink performance of data uploading applications, with saturated uplink traffic.

Uplink is susceptible to the contention of medium access, while the downlink traffic can be coordinated by the AP to avoid contention [10]. If the upper layer protocol overhead is ignored, the download performance for each vehicle can be evenly allocated by the AP [5]. The upload performance, however, is heavily affected by the contention overhead of the uplink. Such contention and proneness to missing control messages have made centralized scheduling schemes difficult to apply to the uplink traffic [11]. The speed limit and traffic conditions in a highway or urban scenario further complicate the analytical model: the number of contending vehicles covered by an AP depends on the vehicle density, and their drive-thru time under the coverage of AP is dependent on the vehicle speed, which is again determined by the vehicle density (e.g., vehicles travel slower on congested roads). The difficulty of uplink scheduling due to time-varying contention, the high mobility of vehicles, and the intermittent connectivity to the infrastructure have made the uplink capacity a nontrivial function of the number of contending vehicles. The analytical modeling in the uplink thus becomes a very challenging task.

All these have motivated us to conduct a careful study on the performance of the uplink, using the IEEE 802.11 distributed coordination function (DCF) medium access control (MAC) protocol that is most widely used for wireless Internet access. The wireless access from vehicles to the AP is much less provisioned when compared with Ethernet or other longhaul access network technologies. Applications are subject to performance degradation due to the high contention among 
vehicles, the varying wireless access delay, and the dynamics of vehicle arrivals and departures. In addition, the deployment and maintenance cost on the infrastructure side can be expensive, so the last hop connectivity can be intermittent. Being the critical and vulnerable component in the entire system, the last-hop access to roadside communication infrastructures from traveling vehicles is thus the focus of our study. Similar uplink scenario also appeared in [22]. The major challenges in this scenario are both in space, i.e., the number of vehicles that can lead to channel contention is constantly changing, and in time, i.e., vehicles only have limited opportunities to upload the data that are in a significant amount.

In this paper, we develop an analytical framework to quantify the uplink performance for a drive-thru Internet using an IEEE 802.11-like contention-based carrier sense multiple access with collision avoidance (CSMA/CA) MAC. Our model is able to capture the performance metrics of a contention-based MAC layer, such as packet collisions, the achievable uplink capacity, etc., given the vehicle density. Meanwhile, for service providers to offer new services in a drive-thru Internet and maintain an optimal and consistent system performance, we propose to adjust the transmission power of AP as a means of admission control. The accuracy of our analytical model and the efficacy of the AP admission control scheme have been validated by the simulation conducted in ns-2. The analytical and simulation results not only have demonstrated the wide applicability of our model, but also have shown the intrinsic nonmonotonic relationship between traffic statistics and uplink performance.

The contributions of this paper are threefold. First, we propose a model for the analysis of the uplink performance of contention-based CSMA/CA MAC with saturated traffic, which is a more challenging problem than that of the downlink. Second, the proposed admission control scheme can help the AP maintain a high performance in the optimal operating region, without any modification at each vehicle. This is critical for service providers to plan and dimension their service provisioning properly, for a consistent user experience. Last, but not the least, our analytical model has been validated through extensive simulation in ns-2.

The rest of this paper is organized as follows. In Section II, we briefly review the capacity analysis of IEEE 802.11 technologies, their applications in vehicular networks, and related work on uplink and downlink performance studies. In Section III, we present our model and analyze the uplink performance metrics given the vehicle density. Analytical and simulation results are compared in Section IV. Further discussions are presented in Section V, and Section VI concludes the paper with future work.

\section{BACKGROUND AND RELATED WORK}

In this section, we present the related work on the performance analysis of IEEE 802.11 CSMA/CA-based MAC in a vehicular network scenario.

\section{A. IEEE 802.11 Performance Analysis}

The performance of IEEE 802.11 in a static wireless local area network (WLAN) has been extensively studied.
Bianchi [3] developed a bidimensional discrete-time Markovchain model to obtain the system throughput of the IEEE 802.11 DCF as a function of the number of saturated contending nodes, with infinite retry limit and perfect channel conditions. Analytical techniques other than Markov-chain models have also appeared, notably the equilibrium point analysis and the mean value analysis. Unlike Bianchi's model, which focuses on microscopic behaviors, such as the transition between backoff counter values and stages, these macroscopic techniques analyze the node contention, transmission, and frame service cycle at a higher level [18]-[20].

In this paper, we follow the renewal reward theory, which is another macroscopic approach, to obtain the analytical results for important system performance metrics in steady state.

\section{B. IEEE 802.11 Performance in Vehicular Networks}

Vehicle-to-infrastructure communications occur in a WLAN with dynamic vehicle movement. Early measurement results from [1] showed that only within a certain distance from the AP, the throughput of vehicles to the AP increases drastically and enters a short "production phase." Eriksson et al. [2] designed and implemented the Cabernet system, and their measurement results show that the network connectivity in such a system is both fleeting and intermittent.

In addition to system and measurement studies, analytical modeling of the performance in drive-thru Internet scenarios has appeared recently. An et al. [4] estimated the collision probability in a vehicular environment, assuming that vehicles are uniformly distributed on road and vehicle speed does not affect vehicle distribution. Alasmary and Zhuang [14] proposed two dynamic contention window mechanisms to alleviate the performance degradation due to the high mobility in vehicular ad hoc networks (VANETs). They also modeled the backoff procedure of IEEE 802.11 as $p$-persistent CSMA/CA, whereas in the standard protocols, the backoff interval is binary exponential.

In this paper, our analytical model follows IEEE 802.11 MAC closely by modeling packet transmissions as a renewal reward process [19]. This model is based on the realistic vehicular traffic characteristics by considering the arrival of vehicles as a random process. The latter has been used in platoon theory [12] and verified by the statistical analysis of empirical data, e.g., [5] and [13]. Furthermore, by following the observed relationship between the vehicle density and the traveling speed according to the traffic flow law [16], we study the impact of traffic conditions on the network performance.

\section{Downlink/Uplink Performance in a Drive-Thru Internet}

In a recent work, Tan et al. [5] investigated the data communication performance in a drive-thru Internet by analyzing the stochastic vehicle arrivals and departures, and by mapping the data download process to the state transition of a series of Markov reward processes. Tan et al. [5] also characterized the per-vehicle capacity, in the presence of other vehicles that equally share the downlink capacity of the AP without extra overhead. Tan et al. [5] studied downlink only, without 


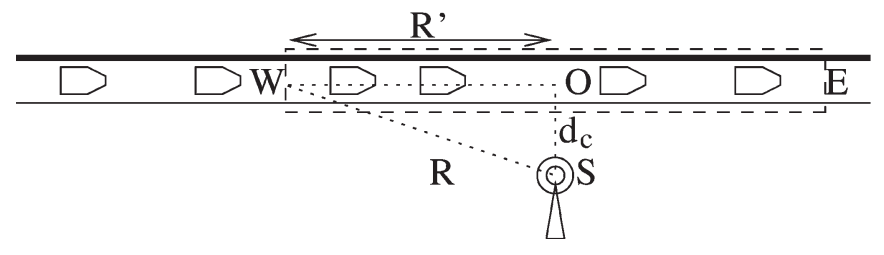

Fig. 1. Network topology and AP placement.

considering the collisions due to random medium access, which affects the overall system performance should there exist uplink traffic. With the contention overhead captured in this work, our model can be degenerated to the scenarios in [5]. We thus focus on the uplink capacity model. When considering both the uplink and downlink sharing the same physical channel, it is equivalent to adding one more contending station, i.e., the AP.

\section{AnAlyticAl Model}

In this section, we present our analytical model for the lasthop access between vehicles and the AP for upload applications with saturated uplink traffic. Considering a roadside AP deployed along a straight road segment as shown in Fig. 1, a typical network setup along a highway or street block. The AP, with a transmission range $R$, is placed slightly off the road by a distance of $|O S|$, thus covering the W-E region of the highway segment of length $2 R^{\prime}=2 \sqrt{R^{2}-|O S|^{2}}$. Users in moving vehicles are able to connect to this AP for Internet access for a certain period of time, as the vehicles pass through its coverage region. Based on the platoon theory [12] and measurement studies [5], [13], the arrival process of vehicles on a highway can be modeled as a Poisson process with rate $\lambda$ (in vehicles per meter).

\section{A. Number of Contending Vehicles}

The number of contending vehicles under the coverage range of AP $n$ is a random variable. Since intervehicle distances are exponentially distributed with mean $1 / \lambda$, the probability that there are $n$ vehicles among the highway segment and within the transmission range $R$ of the AP can be calculated according to the following Poisson distribution:

$$
\operatorname{Pr}(n)=e^{-2 R^{\prime} \lambda}\left(2 R^{\prime} \lambda\right)^{n} / n ! .
$$

Let $q$ be the number of vehicles passing a fixed roadside observation point per second and $v$ be the vehicle speed in meters per second. Together with $\lambda$, these three variables are related by the following fundamental traffic flow law [21]: $q=\lambda v$.

The speed-flow-density diagram from [21] is given in Fig. 2, where a zero flow $(q=0)$ occurs under the following two conditions: 1$)$ when density is zero $(\lambda=0)$ and there are no vehicles on the road or 2) when $\lambda$ reaches the jam density and all vehicles stop. Between these two extreme points, the following linear relationship between speed $v$ and density $\lambda$ is commonly assumed [16]:

$$
v=v_{f}\left(1-\lambda / \lambda_{\mathrm{jam}}\right)
$$

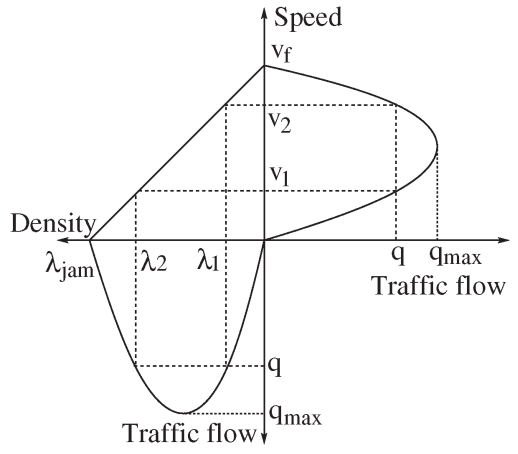

Fig. 2. Relationships between the speed-flow, speed-density, and flow-density [21].

where $v_{f}$ is the free-flow speed that is usually equal to the road speed limit, and $\lambda_{\text {jam }}$ is the vehicle jam density at which traffic flow comes to a halt. $C=\left\lfloor 2 R^{\prime} \lambda_{\text {jam }}\right\rfloor$ is the maximum number of vehicles under the AP coverage. For multiple lanes, the aggregated vehicle arrival rate still follows a Poisson distribution, with $\lambda=\sum \lambda_{i}$, where $\lambda_{i}$ is the vehicle arrival rate on each lane, and lanes are parallel to each other.

\section{B. Conditional Collision Probability}

The channel time is divided into discrete generic slots, a timescale that is much smaller than vehicle arrivals and departures. These generic slots have different lengths, corresponding to the specific channel status of being idle, having a successful transmission or collision. We analyze the system in a steady state, when the contention experienced by a vehicle is dependent on the statistical properties of the vehicle arrival process, rather than the transient behavior of a specific vehicle. An ideal wireless channel without transmission errors is assumed first; therefore, the transmitted frames may be lost only due to the collisions by simultaneous transmissions (channel impairments and other realistic factors will be considered in Section V-A). With IEEE 802.11 DCF, the channel access process of an individual vehicle is regenerative with respect to the time instant of the completion of each successful frame transmission. Therefore, the time period between two consecutive, successful transmissions from the same vehicle form a renewal cycle in a renewal process [19].

Suppose there are $n$ contending vehicles within the coverage of the AP, and let $\tau_{n}$ be the transmission probability of a vehicle in a given generic time slot. $R_{n}$ is the number of transmission attempts of a vehicle for a specific data frame, in the unit of time slots when a transmission or retransmission occurs for this frame. $B_{n}$ is the number of backoff slots during the same transmission cycle. Different from the existing work [18], [19], the challenge here is that $n$ is a random variable, as shown in (1), rather than a fixed number in a typical WLAN.

Denote $E\left[R_{n}\right]$ and $E\left[B_{n}\right]$ as the expectation of $R_{n}$ and $B_{n}$. In each cycle, a vehicle has to go through $E\left[R_{n}\right]+$ $E\left[B_{n}\right]$ generic slots before having one successful transmission. By treating the transmission slots as rewards in the renewal process, with $E\left[R_{n}\right]$ rewards earned every cycle, the long-run 
rate at which the rewards are earned by a vehicle is its transmission probability in a randomly chosen slot, i.e.,

$$
\tau_{n}=E\left[R_{n}\right] /\left(E\left[R_{n}\right]+E\left[B_{n}\right]\right)
$$

where $R_{n}$ follows a truncated geometric distribution, with the following expectation:

$$
E\left[R_{n}\right]=\sum_{i=1}^{m^{\prime}-1} i p_{n}^{i-1}\left(1-p_{n}\right)+m^{\prime} p_{n}^{m^{\prime}-1}
$$

and $p_{n}$ is the conditional collision probability. The expectation of $B_{n}$ is

$$
\begin{aligned}
& E\left[B_{n}\right]=\sum_{i=1}^{m} p_{n}^{i-1}\left(1-p_{n}\right)\left(\sum_{j=1}^{i} b_{j}\right)+p_{n}^{m}\left(1-p_{n}\right) \\
& \quad \times\left(\sum_{j=1}^{m} b_{j}+b_{m}\right)+\cdots+p_{n}^{m^{\prime}-1}\left[\sum_{j=1}^{m} b_{j}+\left(m^{\prime}-m\right) b_{m}\right]
\end{aligned}
$$

where $m$ is the maximum backoff stage, $m^{\prime}$ is the maximum retry limit, and $b_{j}$ is the average value of the backoff counter at backoff stage $j$. Denote the minimum contention window size by $W$; then, $b_{j}=(W / 2) 2^{j-1}$. Both the time lengths of each $R_{n}$ and $B_{n}$ slot in (3)-(5) are multiples of the fixed time slots in IEEE 802.11 standard, which can be of different time durations but in the same order of microseconds. In the generic IEEE 802.11 standard, we have $m=6, m^{\prime}=7$, and $W=32$.

If a vehicle transmits in a given time slot, a collision occurs if and only if at least one of the other $n-1$ vehicles also transmits in the same slot. When $n \geq 1$, we have

$$
p_{n}=1-\left(1-\tau_{n}\right)^{n-1}
$$

The above model captures the contention behavior in the order of several hundred microseconds, while the number of contending vehicles can be assumed constant up to hundreds of milliseconds. In the case where the system is in the steady state, we can ignore the transient behaviors of a specific vehicle entering or leaving the AP coverage. When all vehicles are statistically equivalent to each other, the overall collision probability can be calculated as

$$
E[p]=\frac{\sum_{n=1}^{C} p_{n} \operatorname{Pr}(n)}{\sum_{n=1}^{C} \operatorname{Pr}(n)}
$$

where the summation on the denominator is to normalize the truncated Poisson distribution given $1 \leq n \leq C$. Note that given the distribution of $n$, (7) provides a stochastic average of the conditional collision probability. From (3)-(7), because of the nonlinear relationship between the collision probability and the number of contending vehicles, the steady-state performance given a stochastic vehicle arrival process is not the performance with the average number of vehicles of this arrival process. Thus, obtaining $E[p]$ by simply replacing $n$ with $E[n]$ in the existing results does not hold.

\section{Frame Service Time}

The channel access procedure of a tagged vehicle regenerates itself for each frame. Therefore, the length of a renewal cycle is essentially the frame service time, i.e., the complete service period for each MAC frame. Assume that there are $n$ vehicles under the coverage of the AP. Denote that in a generic time slot, the stationary probability of the wireless channel being idle as $a_{n}$, and the probability of having a successful or collided transmission as $b_{n}$ and $c_{n}$, respectively, we have

$a_{n}=\left(1-\tau_{n}\right)^{n}, \quad b_{n}=n \tau\left(1-\tau_{n}\right)^{n-1}, \quad c_{n}=1-a_{n}-b_{n}$.

Let $T_{a}, T_{b}$, and $T_{c}$ be the time duration of a generic slot being idle, having a successful transmission and collision, respectively. $T_{a}$ equals the fixed slot time $\delta$, which is given in the IEEE 802.11 standard. In the basic access mode of 802.11 DCF, a successful frame transmission includes the transmission of the DATA frame, the ACK from the receiver, and the short interframe space (SIFS) in between. Also, the backoff timer only resumes after the channel has been idle for DCF interframe space (DIFS), so $T_{b}=T_{\mathrm{DATA}}+T_{\mathrm{SIFS}}+T_{\mathrm{ACK}}+T_{\mathrm{DIFS}}$. On the other hand, a vehicle that detects a collision will resume its backoff timer after EIFS $=T_{\mathrm{SIFS}}+T_{\mathrm{ACK}}+T_{\mathrm{DIFS}}$, and therefore, $T_{c}=T_{\mathrm{DATA}_{\max }}+T_{\mathrm{SIFS}}+T_{\mathrm{ACK}}+T_{\mathrm{DIFS}}$, where DATA $_{\max }$ is the longest data frame incurred in the collision. If we assume that all data frames have the same size, then $T_{b}=T_{c}$. The detailed computation of $T_{\mathrm{DATA}}$ and $T_{\mathrm{ACK}}$ will be discussed in Section IV. Given the above notations, the average duration of a generic time slot is

$$
E\left[D_{n}\right]=T_{a} a_{n}+T_{b} b_{n}+T_{c} c_{n}
$$

i.e., a statistical average of the channel being idle, successfully transmitting, or in collision. If there are $n$ contending vehicles, the average time to finish transmitting a data frame, or the frame service time, is the product of the number of the generic slots experienced by a frame, and the slot duration in (8), i.e.,

$$
E\left[T_{n}\right]=\left(E\left[R_{n}\right]+E\left[B_{n}\right]\right) E\left[D_{n}\right]
$$

where $E\left[R_{n}\right]$ and $E\left[B_{n}\right]$ are given in (4) and (5), respectively.

\section{Per-Vehicle Throughput}

With the frame service time obtained above, the throughput achieved by each vehicle is

$$
E[\Gamma]=E[P L]\left(1-E[p]^{m^{\prime}}\right) / E[T]
$$

where $P L$ is the payload length of each frame on the application layer, $E[p]$ is given in (7), and

$$
E[T]=\frac{\sum_{n=1}^{C} E\left[T_{n}\right] \operatorname{Pr}(n)}{\sum_{n=1}^{C} \operatorname{Pr}(n)} .
$$

Equation (10) can be explained as follows. Each frame is transmitted at most $m^{\prime}$ times, either successfully or dropped due to reaching the retry limit. As those dropped frames do not contribute to throughput, we only count $E[P L]\left(1-E[p]^{m^{\prime}}\right)$, 
which is the effective part of the payload. Therefore, the throughput is the effective payload length over the average frame service time, i.e., how long to transmit a frame through the network. It is the throughput achieved by any one of the $n$ vehicles if they are statistically equivalent.

\section{E. Network Throughput}

The throughput achieved by each vehicle in (10) is for an individual vehicle. The analysis on network throughput can be simplified from the viewpoint of the AP: the network throughput is the total amount of transmitted data that the AP can receive successfully per unit time, regardless of which vehicles have transmitted them. Given $n$ contending vehicles, the network throughput is the average payload per frame over the average frame service time, i.e.,

$$
\Pi_{n}=E[P L] n \tau_{n}\left(1-\tau_{n}\right)^{n-1} / E\left[D_{n}\right] .
$$

Then, the overall network throughput is $E[\Pi]=$ $\left(\sum_{n=1}^{C} \Pi_{n} \operatorname{Pr}(n)\right) /\left(\sum_{n=0}^{C} \operatorname{Pr}(n)\right)$, where the summation in the denominator starts from $n=0$ to include the probability of the AP being idle.

\section{F. Data Uploaded per Drive-Thru}

The average throughput per vehicle $E[\Gamma]$ decreases with vehicle density $\lambda$ since a larger number of vehicles leads to a higher contention overhead and less capacity to share. However, the total amount of the data uploaded by a vehicle $\Lambda$ is determined by both the throughput $E[\Gamma]$ and the drivethru time $t$. $t$ is determined by the AP's transmission range $R$ and vehicle speed $v$, where the vehicle speed is related to the vehicle density according to (2). As a result, the expectation of $\Lambda$ can ultimately be characterized by a single parameter $\lambda$, i.e.,

$$
E[\Lambda]=E[\Gamma] t=\frac{2 E[\Gamma] R^{\prime}}{v_{f}\left(1-\lambda / \lambda_{\mathrm{jam}}\right)} .
$$

The uploaded data amount in fact is not a monotonic function of vehicle density, which will be demonstrated in Section IV.

\section{G. Approximated Distribution of Uploaded Data}

The distribution of the uploaded data of a vehicle $\operatorname{Pr}\{\Lambda \leq$ $k\}$, is defined as the probability that a vehicle can upload $k$ data frames during its drive-thru time under the AP's coverage $t$. The distribution of $\Lambda$ can be used to analyze the percentage of vehicles that may experience unsatisfactory services, e.g., when $\Lambda$ is below a threshold. In the following, we derive this distribution using a Gaussian approximation.

Since the instantaneous throughput of a vehicle depends on the number of vehicles under the AP coverage $n$, we first derive the conditional probability $\operatorname{Pr}\{\Lambda \leq k \mid n\}$. The probability that $\Lambda$ is no more than $k$ equals the probability that the sum of the frame service time of $k$ frames is no less than $t$. Denote $T_{n, i}$ as the frame service time of the $i$ th frame, given that there are $n$ vehicles. As the value of $k$ is typically a large number $(k \gg 10)$, by the central limit theorem, the sum of $k$ independent identically distributed random variables can be well approximated by a Gaussian random variable $\sum_{i=1}^{k} T_{n, i} \sim$ $\mathcal{N}\left(k E\left[T_{n}\right], k \operatorname{Var}\left(T_{n}\right)\right)$.

Next, we need to find the variance of $T_{n}$, which is the product of two independent random variables, namely, the number of the generic slots experienced by a frame $\left(S_{n}\right)$ and the slot duration $\left(D_{n}\right)$, as in (9). $S_{n}$ can be approximated by a geometric distribution with probability $\tau_{n}\left(1-\tau_{n}\right)^{n-1}$, and therefore, its mean and variance are $E\left[S_{n}\right]=\left(\tau_{n}\left(1-\tau_{n}\right)^{n-1}\right)^{-1}$, and $\operatorname{Var}\left(S_{n}\right)=\left(1-\tau_{n}\left(1-\tau_{n}\right)^{n-1} /\left[\tau_{n}\left(1-\tau_{n}\right)^{n-1}\right]^{2}\right)$. The mean of $D_{n}$ is given in (8), and its variance is $\operatorname{Var}\left(D_{n}\right)=$ $\left(T_{a}-E\left[D_{n}\right]\right)^{2} a_{n}+\left(T_{b}-E\left[D_{n}\right]\right)^{2} b_{n}+\left(T_{c}-E\left[D_{n}\right]\right)^{2} c_{n}$. The variance of $T_{n}\left(=S_{n} D_{n}\right)$ can be obtained by $V \operatorname{ar}\left(T_{n}\right)=$ $\left(E\left[S_{n}\right]\right)^{2} V \operatorname{ar}\left(D_{n}\right)+\left(E\left[D_{n}\right]\right)^{2} V \operatorname{ar}\left(S_{n}\right)+V \operatorname{ar}\left(S_{n}\right) V \operatorname{ar}\left(D_{n}\right)$.

Given the mean and variance of $T_{n}$, we can derive the conditional $\mathrm{CDF}$ of the uploaded data as follows:

$$
\operatorname{Pr}\{\Lambda \leq k \mid n\}=\operatorname{Pr}\left\{\sum_{i=1}^{k} T_{n, i}>t\right\}=Q\left(\frac{t-k E\left[T_{n}\right]}{\sqrt{k \operatorname{Var}\left(T_{n}\right)}}\right)
$$

where $Q(\cdot)$ is the $Q$-function. Since the vehicle arrivals follow a Poisson process, the number of vehicles does not change drastically during a short interval. We can approximate the unconditional $\mathrm{CDF}$ function of the uploaded data as follows:

$$
\operatorname{Pr}\{\Lambda \leq k\}=\frac{\sum_{n=1}^{C} \operatorname{Pr}\{\Lambda \leq k \mid n\} \operatorname{Pr}(n)}{\sum_{n=1}^{C} \operatorname{Pr}(n)} .
$$

\section{Performance Evaluation}

In this section, we provide an extensive evaluation on the analysis through the simulation conducted in ns-2.34.

\section{A. Simulation Model and Parameters}

1) Signal Propagation and AP Placement: Considering the signal propagation in an open area with a small number of reflectors, e.g., highway scenarios, the electromagnetic wave propagation can be modeled as a power-law function of the distance $d$ between transceivers [15]. Given the transmission power $P_{t}$, the received power $P(d)$ as a function of $d$ is

$$
P(d)= \begin{cases}P_{t} G_{t} G_{r} \frac{\lambda_{c}^{2}}{(4 \pi d)^{2} L}, & d_{0} \leq d<d_{c} \\ P_{t} G_{t} G_{r} \frac{h_{t}^{2} h_{r}^{2}}{d^{4}}, & d \geq d_{c}\end{cases}
$$

where $\lambda_{c}$ is the carrier wavelength, $G_{t}$ and $G_{r}$ are the transceiver antenna gains, $h_{t}$ and $h_{r}$ are the transceiver antenna heights, respectively, and $L$ is the system loss factor. The crossover distance is given by $d_{c}=4 \pi \sqrt{L} h_{t} h_{r} / \lambda_{c}$.

The above propagation model characterizes the properties of large-scale path loss at a high level, giving an upper bound of the MAC layer performance, when the signal variation due to the blockage from multiple obstacles (other than ground reflection) is not severe. All the physical layer parameters are listed in Table I(a). The resultant $d_{c}$ is $38.31 \mathrm{~m}$. In addition to the large-scale fading, shadowing and multipath fading can be considered through packet error rate (PER) $p_{e}$. Such channel 
TABLE I

Physical Layer PARAMEters. (a) RF CONFiguration. (b) NS-2 SETTINGS.

(a)

\begin{tabular}{ccc}
\hline Notation & Meaning & Value \\
\hline \hline$P_{t}$ & Transmission power & $0.28183815 \mathrm{~W}$ \\
\hline$G_{t}$ & Gain of the transmitter antenna & 1 \\
\hline$G_{r}$ & Gain of the receiver antenna & 1 \\
\hline$h_{t}$ & Height of the transmitter antenna & $1 \mathrm{~m}$ \\
\hline$h_{r}$ & Height of the receiver antenna & $1 \mathrm{~m}$ \\
\hline$\lambda_{c}$ & Wavelength of the carrier signal & $0.328 \mathrm{~m}^{1}$ \\
\hline$L$ & System loss factor & 1 \\
\hline
\end{tabular}

(b)

\begin{tabular}{lccc}
\hline Notation & Meaning & 802.11 & $802.11 \mathrm{p}$ \\
\hline \hline CPThresh & Capture threshold & $1.6 \mathrm{e} 3$ & 43 \\
\hline CSThresh & Carrier sense threshold & $4.7 \mathrm{e}-12 \mathrm{~W}$ & $1.8 \mathrm{e}-11 \mathrm{~W}$ \\
\hline RXThresh & Receiver threshold & $7.2 \mathrm{e}-11 \mathrm{~W}$ \\
\hline PCLPPLen & PLCP preamble length & \multicolumn{2}{c}{18 bytes } \\
\hline PLCPHLen & PLCP header length & \multicolumn{2}{c}{6 bytes } \\
\hline PLCPDR & PLCP data rate & \multicolumn{2}{c}{$1 \mathrm{Mbps}$} \\
\hline PHYDR & PHY-layer data rate & $1 \mathrm{Mbps}$ & $3 \mathrm{Mbps}$ \\
\hline
\end{tabular}

${ }^{1}$ Using the default $914 \mathrm{MHz}$ radio in $\mathrm{ns}-2, \lambda_{c}=3 \times 10^{8} / 914 \times 10^{6}=$ $0.328 \mathrm{~m}$. Similar approach can be applied to 5.8 or $2.4 \mathrm{GHz}$ IEEE $802.11 \mathrm{a} / \mathrm{b} / \mathrm{g} / \mathrm{n}$ or $5.9 \mathrm{GHz}$ IEEE $802.11 \mathrm{p}$.

impairments may also occur due to interference, mobility, and other nondeterministic factors, which will be discussed in Section V-A. Equation (15) is applicable only in the far-field cases. As we place the AP at a distance $|O S|=d_{c}$ away from the road, the far-field two-ray ground propagation takes effect in this case. This is also realistic as APs are usually installed in rest areas, instead of directly on a highway.

2) NS-2 Threshold Parameters: As shown in Fig. 1, with a fixed transmission power, the strongest signal is received when a vehicle is transmitting at $\mathrm{O}$, while the weakest signal happens when it is at $\mathrm{W}$ or $\mathrm{E}$. To focus on the contention nature of the uplink first, we intentionally set a large capture threshold now so that if the AP is receiving a frame from a vehicle at $\mathrm{O}$, a concurrent transmission from $\mathrm{W}$ or $\mathrm{E}$ (if any) will cause a collision. For IEEE 802.11, we have CPThresh $\geq$ $(P(|O S|) / P(|W S|))=1.6 \times 10^{3}$. The details of handling realistic capture effect and channel impairments are given in Section V-A.

Note that we also need to set the carrier sense (CS) threshold properly. That is, if a vehicle at $\mathrm{W}$ transmits, another vehicle at E can sense that the channel is busy and back off for collision avoidance. For IEEE 802.11, we have CSThresh $\leq$ $P(|W E|)=P\left(2 \sqrt{R^{2}-|O S|^{2}}\right)=4.7 \times 10^{-12} W$. Further, for the AP to correctly receive the frame transmitted by the farthest vehicle at $\mathrm{W}$ or $\mathrm{E}$, the receiver threshold, according to (15), must be RXThresh $\leq P(|W S|)=7.2 \times 10^{-11} W$.

All the ns-2-related settings are listed in Table I(b), including the physical layer convergence protocol (PLCP) preamble and header length, PLCP data rate, and physical data rate, which will be used for the calculation of the duration of a frame transmission and thus a generic time slot. The last column of Table I(b) is for IEEE $802.11 \mathrm{p}$, which has been proposed specifically for VANETs, whose results will be presented in Sections IV-E and F for comparison.

3) Network Synchronization: In the worst case, the propagation delay from $\mathrm{W}$ to $\mathrm{E}$ is $2 \sqrt{|W S|^{2}-|O S|^{2}} / 3 \times 10^{8}=$

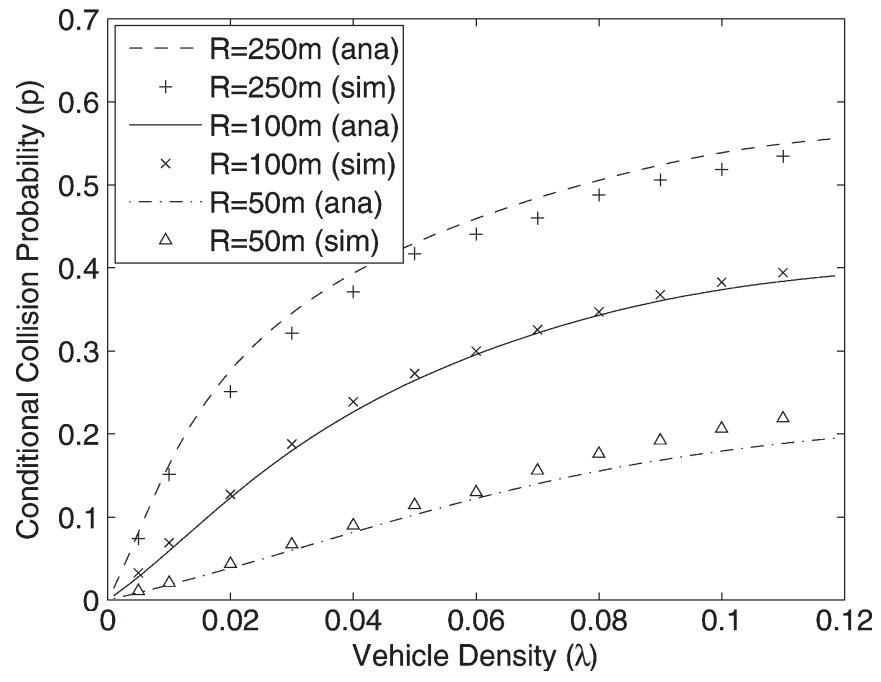

Fig. 3. Collision probability versus vehicle density.

$1.65 \mu \mathrm{s}$. This is much smaller than the fixed slot time $\delta=20 \mu \mathrm{s}$, and thus, the entire network can be assumed synchronized. Section $\mathrm{V}$ will discuss how to take the hidden terminal and other practical issues into account.

\section{B. Collision Probability}

We consider an IEEE-802.11-based AP with transmission range $R=250,100$, and $50 \mathrm{~m}$, which are the typical communication ranges of IEEE 802.11 APs. Fig. 3 shows the analytical results according to (7) and the simulation results obtained using ns- 2 by averaging over 20 runs. Obviously, as the vehicle density increases, more vehicles are under the AP coverage, and the uplink collision probability increases. Also, a smaller transmission range reduces the collision probability, due to fewer contending vehicles. From the figure, our model can characterize the collision probability accurately by considering the vehicle statistics.

\section{Per-Vehicle Throughput}

In (10), we need to compute the data frame and acknowledgment transmission time, $T_{\mathrm{DATA}}$ and $T_{\mathrm{ACK}}$. Payload will be encapsulated with 58-B packet headers from the lower layers. However, for each frame, a fixed-length PLCP preamble and PLCP header are transmitted at a fixed data rate (which may be different from the data transmission rate) [17]. Supposing the average application layer data payload length per frame is $E[P L]$ bytes, then the total time to transmit the physical layer frame is the time to send the PLCP preamble and header at the PLCP data rate, plus the time to send the PLCP payload at the chosen physical layer data rate: TXTIME $=($ PCLP $\times$ $8 /$ PLCPDR $)+((E[P L]+58-$ PCLP $) \times 8 /$ PHYDR $)$, where PCLP $=$ PCLPPLen + PLCPHLen.

In addition to the PHY and MAC layer parameters in Tables I and II, we use the following parameters at the application layer: $E[P L]=1000 \mathrm{~B} /$ packet as the fixed payload length and application data rate at $875 \mathrm{~kb} / \mathrm{s}$. The analytical model presented in Section III is applicable to any saturated traffic, 
TABLE II

MAC LAYER PARAMETERS

\begin{tabular}{cccc}
\hline Notation & Meaning & 802.11 & $802.11 \mathrm{p}$ \\
\hline \hline$T_{a}$ & Time duration of an idle slot & \multicolumn{2}{c}{$\delta$} \\
\hline$T_{b}$ & $\begin{array}{c}\text { Time duration of } \\
\text { a successful transmission }\end{array}$ & \multicolumn{2}{c}{$T_{\text {DATA }}+T_{\text {SIFS }}+T_{\text {ACK }}+T_{\text {DIFS }}$} \\
\hline$T_{c}$ & Time duration of collision & \multicolumn{2}{c}{$=T_{b}$} \\
\hline$\delta$ & Slot time & $20 \mu \mathrm{s}$ & $13 \mu \mathrm{s}$ \\
\hline$T_{\text {SIFS }}$ & SIFS time & $10 \mu \mathrm{s}$ & $32 \mu \mathrm{s}$ \\
\hline$T_{\text {DIFS }}$ & DIFS time & $50 \mu \mathrm{s}$ & $58 \mu \mathrm{s}$ \\
\hline$T_{\text {DATA }}$ & $1 K B$ data transmission time & $8,464 \mu \mathrm{s}$ & $2,949 \mu \mathrm{s}$ \\
\hline$T_{\mathrm{ACK}}$ & ACK transmission time & $304 \mu \mathrm{s}$ & $229 \mu \mathrm{s}$ \\
\hline CWMin & Minimum backoff window & 32 & 16 \\
\hline CWMax & Maximum backoff window & 1024 & 1024 \\
\hline$m$ & Maximum backoff stage & 6 & 7 \\
\hline$m^{\prime}$ & Maximum retry limit & 7 & 7 \\
\hline
\end{tabular}

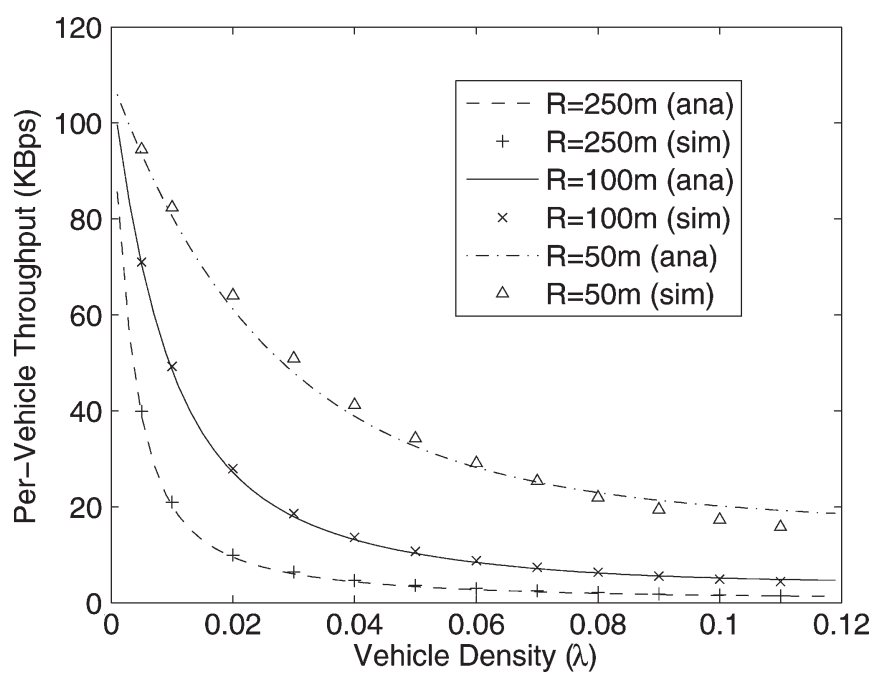

Fig. 4. Per-vehicle throughput versus vehicle density.

and the constant bit rate is used as an example in this section. We also assume that penetration rate is $100 \%$, i.e., all vehicles are equipped with IEEE 802.11 devices. The transmission time $T_{\mathrm{DATA}}$ and $T_{\mathrm{ACK}}$ in (8) can thus be calculated accordingly, which are both listed in Table II, with the physical layer data rate at $1 \mathrm{Mb} / \mathrm{s}$ for the generic IEEE 802.11. Throughput analysis and simulation results are given in Fig. 4. From the figure, we can tell that our renewal reward theory-based modeling and analysis approach captures the throughput metric accurately.

By considering Fig. 3, collision will occur more frequently when vehicle density is high. In Fig. 4, vehicle throughput is monotonically decreasing with respect to an increasing traffic density. Note that under extreme circumstances when the contention is very severe, e.g., $\lambda>0.1$, it is difficult for vehicles to reach a steady state. As a result, the analytical results are less accurate in these cases. Also, $R=100$ and $50 \mathrm{~m}$ have a higher per-vehicle throughput than $R=250 \mathrm{~m}$ for all vehicle densities. This individual performance gain, however, is at a high system cost: With a shorter transmission range $R$, fewer vehicles are able to communicate with the AP because of the intermittent connectivity [1], [2]. A direct consequence is the limited service time in total, which has an impact on the amount of the overall data uploaded by vehicles.

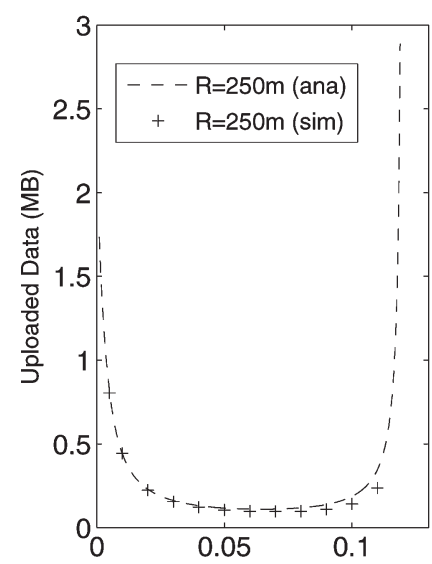

(a)

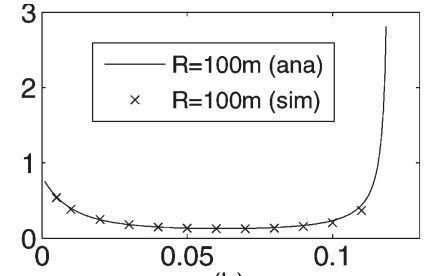

(b)

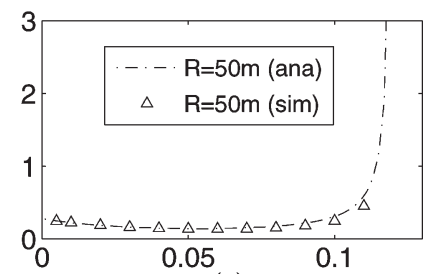

(c)
Fig. 5. Data uploaded per drive-thru.

\section{Uploaded Data per Drive-Thru}

The amount of the data uploaded by a vehicle when driving through a roadside AP is of great importance. According to [5], a typical highway vehicle jam density $\lambda_{\text {jam }}$ is 200 vehicles $/ \mathrm{mi}$, which is 0.12 vehicles $/ \mathrm{m}$, and a common free flow speed $v_{f}=55 \mathrm{mi} / \mathrm{h} \approx 24.59 \mathrm{~m} / \mathrm{s}$. Vehicle speed under different densities can be estimated by (2). Analytical and simulation results are given in Fig. 5(a)-(c) for $R=250,100$, and $50 \mathrm{~m}$, respectively, showing the amount of data uploaded by each vehicle during their drive-thru interval. The results demonstrate a nonmonotonic behavior with respect to the vehicle density $\lambda$.

From the figure, at low vehicle densities, which correspond to higher vehicle speeds, there are very few vehicles and, thus, lower contention for the uplink communication. Although the connectivity duration with the AP is short, vehicles are able to upload at a high throughput. As the vehicle density increases, contention increases quickly, thus leading to a much higher collision, as we have seen in Fig. 3. The amount of the uploaded data at this level drastically decreases even though the vehicles are traveling at a lower speed with a longer drive-thru time. This corresponds to an undesirable data transmission stage with both a low throughput and high collision. Thus, when the traffic density is in a low-to-medium range, the number of contending vehicles under the AP coverage, or the coverage range, determines the network performance.

When the vehicle density increases further to the jam density, the amount of the uploaded data will approach infinity, as the vehicle suffers from congestion and eventually comes to a stop. However, with a very high vehicle density, the achievable throughput per vehicle in Fig. 4 is very low, whereas most Internet applications require a minimum sustainable throughput. In addition, the ultrahigh vehicle density leads to a very low vehicle speed, and vehicles have to wait a much longer time until they can reach the coverage range of the next AP. Thus, maintaining a high upload throughput and achieving a large amount of uploaded data at the same time is the goal of the optimal operating region in drive-thru Internet scenarios.

While the shape of Fig. 5(a)-(c) is similar to the results in [5], this is only due to the fundamental traffic flow law shown 


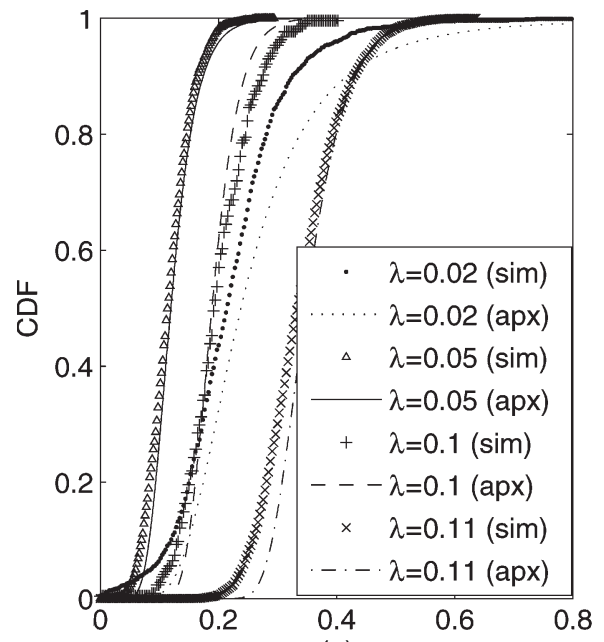

(a)

Fig. 6. Distribution of the uploaded data $(R=250 \mathrm{~m})$.

in Fig. 2 (i.e., vehicles travel slowly on congested roads). The model in [5] analyzed the downlink capacity that is equally shared among vehicles, and the corresponding results cannot predict the performance when vehicles need to compete for the uplink using a random access MAC protocol.

\section{E. Distribution of Uploaded Data}

In this section, we illustrate the distribution of the amount of uploaded data for several representative vehicle densities, with both the generic IEEE 802.11 and IEEE 802.11p that is tailored for vehicular networks. The IEEE $802.11 \mathrm{p}$ standard works at $5.9 \mathrm{GHz}$ frequency, resulting in a carrier wavelength $\lambda_{c}=0.0508 \mathrm{~m}$. The physical layer configurations are listed in Table I(b), assuming a $10-\mathrm{MHz}$ channel bandwidth with a physical layer data rate of $3 \mathrm{Mb} / \mathrm{s}$. In IEEE $802.11 \mathrm{p}$, the MAC layer parameters such as the slot time, frame space times, and, thus, the length of a generic time slot are also different. The corresponding settings are in Table II. $E[P L]$ is also $1000 \mathrm{~B} /$ packet, and the application data rate is $2500 \mathrm{~kb} / \mathrm{s}$ given a higher data rate at the physical layer.

Fig. 6 shows the uploaded data distribution collected from ns-2 traces and our approximation in (14), with high, mid, and low vehicle densities. The figure shows that the approximation can match the simulation trace reasonably well, and the uploaded data at $3 \mathrm{Mb} / \mathrm{s}$ in IEEE $802.11 \mathrm{p}$ is almost three times as that of $1 \mathrm{Mb} / \mathrm{s}$ in the generic IEEE 802.11. With the uploaded data distribution, the service provider can estimate the probability that the data uploaded by each drive-thru vehicle is above a given threshold, which is important for quality of service provisioning and user experience guarantee. The deviation between the approximation and ns- 2 trace at very low density (i.e., $\lambda=0.02$ ) is due to the short drive-thru time that does not allow the system to become stable enough.

Tan et al. [5] also obtained the distribution of the transferred data, but only for the collision-free downlink, and their model is very computationally expensive. Our Gaussian approximation and the ns-2 trace show that our approach is both simple and reasonably accurate.

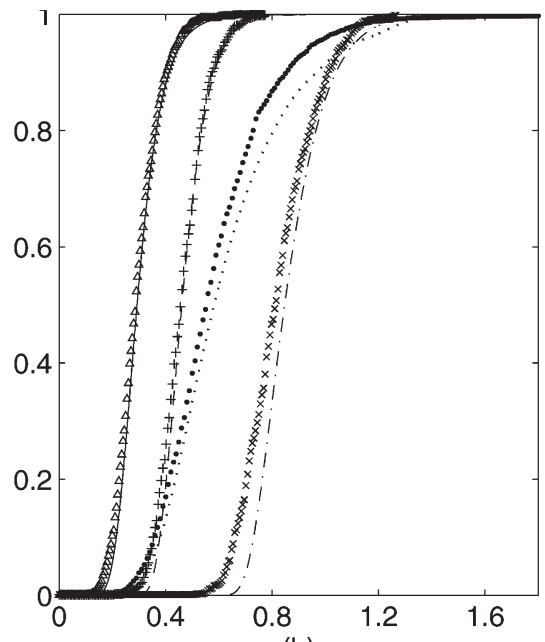

(b)

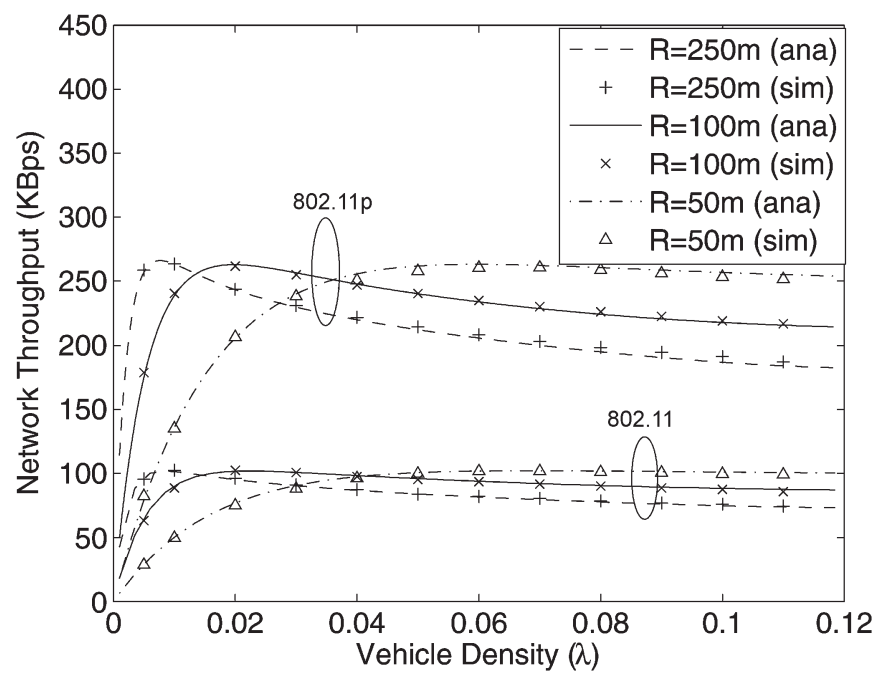

Fig. 7. Network throughput versus vehicle density.

\section{F. Network Throughput and Admission Control}

We assume that vehicles use fixed transmission power, so the packets from the vehicles within the AP coverage can always reach the AP. The AP determines whether to lower its transmission power, depending on the current vehicle density and the resultant contention intensity so that the coverage of its beacon messages is adjusted accordingly for admission control.

Knowing the optimal operating region that has both a high throughput and a large amount of uploaded data, we explore the adjustment of transmission power as a means of admission control by the AP. Fig. 7 shows the analytical and simulation results for network throughput, where the analysis is very accurate when compared with the simulation for the entire network. Notice that for all the cases in both the generic IEEE 802.11 and $802.11 \mathrm{p}, R=250,100$, and $50 \mathrm{~m}$, there is an optimal region where the network throughput is maximized. For example, at very low vehicle densities, i.e., $\lambda \leq 0.01, R=250 \mathrm{~m}$ gives the highest throughput. This is because vehicle contention is low at these densities, and using a large transmission range can reduce the AP idle periods. The same observation can be made from 


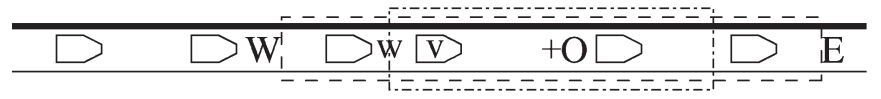

Fig. 8. Capture effect and model extension.

Fig. 5, where $\lambda \leq 0.01$ and $R=250 \mathrm{~m}$ also leads to a large amount of uploaded data. When $\lambda$ increases, i.e., $0.01<\lambda \leq$ 0.04 , using a smaller transmission range $R=100 \mathrm{~m}$ still keeps the network throughput at a high level, while continuing with $R=250 \mathrm{~m}$ will reduce the network throughput significantly. When $\lambda>0.04, R=50 \mathrm{~m}$ becomes the optimal transmission range for AP to avoid severe transmission collisions. From Fig. 7, the network throughput in IEEE $802.11 \mathrm{p}$ is also almost three times as that in the generic IEEE 802.11. On the other hand, the optimal operating regions of the AP in both cases are quite similar.

The AP can reduce its transmission range by reducing transmission power; or if the vehicles are cooperative, the AP can reduce the transmission range by using a high-data-rate modulation and coding scheme in the physical layer with the same transmission power. For example, IEEE $802.11 \mathrm{~b}$ supports 1, 2, 5.5 , and $11 \mathrm{Mb} / \mathrm{s}$ at different distances. When only admitting vehicles in close proximity to the AP, vehicles will be able to transmit at a higher data rate, e.g., $11 \mathrm{Mb} / \mathrm{s}$ when $R=50 \mathrm{~m}$ and $2 \mathrm{Mb} / \mathrm{s}$ when $R=100 \mathrm{~m}$, which will increase the system capacity and vehicle throughput, and eventually, the amount of uploaded data. However, reducing transmission range also has other consequences, e.g., it takes a longer time for vehicles to reach the coverage range of an AP, and more APs have to be deployed, which increases the system cost. We leave the full exploration of this scheme, i.e., adaptive data rate at different transmission ranges of APs, for future work.

\section{FURTHER DisCUSSIONS}

We have made certain assumptions and simplifications in analysis and simulation, and here, we discuss their implication and how we can mitigate their impact.

\section{A. Capture Effect and Channel Impairments}

1) Capture Effect: In the analytical model and simulation, we have ignored the capture effect. In reality, when two or more frames collide at the receiver, the one with the strongest signal strength and above the capture effect threshold with regard to the other frames, still can be received and decoded properly. We can take capture effect into account in our model by letting $p_{n}$, the conditional collision probability given there are $n$ contending vehicles, become location dependent, i.e., $p_{n}(d)$, where $d$ is the distance from the tagged vehicle ( $v$ in Fig. 8 ) to the AP ( $S$ in Fig. 1). Due to the capture effect, if any vehicles within $d$ transmit in the same generic slot, the tagged vehicle has a collision deterministically. For the vehicles of distance $D$ away from the $\mathrm{AP}$, if $(D / d)^{\alpha} \geq \mathrm{CPThresh}$, where $\alpha$ is the path-loss exponent, those vehicles will not cause any collisions for the tagged vehicle even they are transmitting in the same generic slot (e.g., the vehicles in the range of $[W, w]$ in Fig. 8). Thus, for a vehicle that is $d$ meters away from the AP, the number of

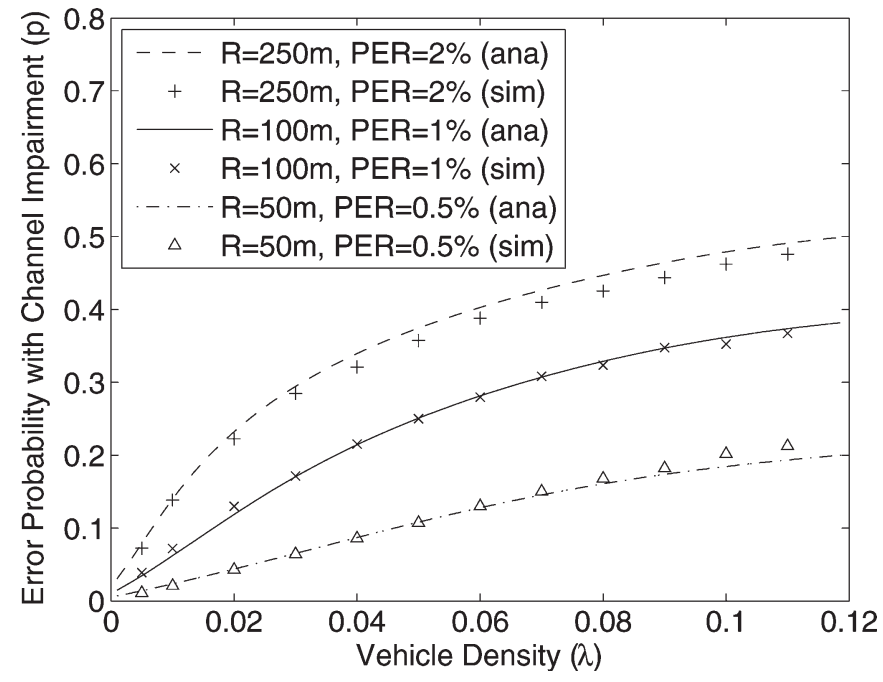

Fig. 9. Error probability with capture effect and channel impairments.

contending vehicles becomes $n=2 \lambda \times \min \left\{R^{\prime}, \sqrt{D^{2}-d_{c}^{2}}\right\}$. Since vehicles travel through the coverage range of AP, they will be at disadvantage or at advantage due to capture effect in a statistically equivalent way, and the capture effect only effectively reduces the collision probability seen by all vehicles, as $\min \left\{R^{\prime}, \sqrt{D^{2}-d_{c}^{2}}\right\} \leq R^{\prime}$.

2) Channel Impairments: In the previous sections, we have focused on path loss in radio propagation, and assumed that a receiver can successfully receive all the successfully transmitted packets. With the consideration of channel impairments, packet errors will be introduced by shadowing, multipath fading, interference, etc. On a highway, the Doppler fading due to the high vehicle speed is typically a dominating factor. From the physical to upper layers, there are several techniques to deal with transmission errors caused by imperfect channel conditions. For example, the MAC layer retransmission is an efficient way to correct the corrupted frames; therefore, our model can be easily extended to consider MAC layer retransmissions due to transmission errors. Under a realistic channel condition, the transmission errors can be caused by a collision probability with capture effect $\left(p_{n}(d)\right.$ in Section V-A1), as well as a packet transmission error probability $p_{e}$ due to shadowing, multipath fading, etc. Therefore, the total error probability is $p=1-\left(1-p_{n}(d)\right)\left(1-p_{e}\right)$, which can be used in the model built in Section III.

Combining capture effect and channel impairments with the uplink performance analysis in Section III, the results are shown in Fig. 9, with the default capture threshold CPThresh = 10 in ns-2, and a PER of $2 \%, 1 \%$, and $0.5 \%$, respectively. From the figure, our analytical model is still accurate when considering the capture effect and channel impairments. With an accurately predicted error probability in the extended model, other performance metrics can be obtained, since they all essentially rely on the varying packet error probability.

\section{B. Nonsaturated Traffic and Multiple APs}

In Section IV, UDP and saturated uplink traffic are assumed, which is the case for bulk data upload applications. With 
nonsaturated data sources, the traffic arrival rate is less than the service rate. As a result, the long-term throughput is equal to the actual data arrival rate. The analytical model for saturated data sources therefore gives an upper bound on the system capacity when the data sources are persistent, and nonpersistent ones actually reduce the perceived contention. Meanwhile, as a side effect of using UDP, the delay of the upper layer connection establishment has been ignored. However, compared with the long session time of the bulk data transfer, the overhead of TCP handshake and connection establishment is relatively constant and of short duration and can be amortized during the entire bulk data transfer session. The impact of nonsaturated traffic and reliable transport protocols, such as TCP, remains an open issue for our following work.

We also have focused on the case where a single AP is deployed along a road segment. How to deploy multiple APs to provide intermittent Internet access for the people on the move needs more careful planning. Dense deployment can improve the coverage of traveling vehicles, but involves a higher system cost. Considering the total uploaded data versus the total traveling time for a vehicle moving from one AP to another, the optimal deployment problem needs further investigation.

\section{Hidden Terminals}

Hidden terminals can be effectively eliminated with short request to send/clear to send (RTS/CTS) messages. However, the IEEE 802.11 basic mode and IEEE 802.11p do not use RTS/CTS due to the associated overhead. Unlike capture effect, hidden terminal does affect the MAC performance adversely. For modern wireless hardware, the carrier sensing range is at least twice of the reliable transmission range, and in the drive-thru Internet scenarios, this can virtually eliminate hidden terminals. Even if the farthest vehicles cannot decode the frame properly to set their NAV vectors in the logical CS procedure, they still can realize that the channel is busy and backoff properly due to the physical CS procedure. Drive-thru Internet has limited coverage and typically uses single-hop transmissions, so the propagation delay is very small. Thus, hidden terminals do not have noticeable impact on the performance metrics of our interest. However, if vehicles relay data for each other, hidden terminals may play a role in the system performance, which requires further investigation.

\section{CONCLUSION}

In this paper, we have proposed an analytical framework to obtain the upload performance metrics for a drive-thru Internet. We have considered different vehicle densities and the corresponding traveling speed, as well as different transmission ranges of the AP. The analytical model has been validated by extensive simulation conducted in the open-source simulator ns-2. Furthermore, we have proposed and investigated the efficacy of admission control by the AP through transmission power control to maintain an optimal system performance for a wide range of vehicle densities. As the drive-thru Internet and other similar opportunistic networking scenarios have attracted increasingly more attention from the industry, we believe this work will help service providers to properly plan and dimension their service infrastructure and offerings and to provide a consistent user experience to customers.

\section{ACKNOWLEDGMENT}

Y. Zhuang would like to thank R. Ruby for the help on ns-2.

\section{REFERENCES}

[1] J. Ott and D. Kutscher, "Drive-thru Internet: IEEE $802.11 \mathrm{~b}$ for 'Automobile' Users," in Proc. IEEE INFOCOM, 2004, pp. 1-12.

[2] J. Eriksson, H. Balakrishnan, and S. Madden, "Cabernet: Vehicular content delivery using WiFi," in Proc. ACM MobiCom, 2008, pp. 199-210.

[3] G. Bianchi, "Performance analysis of the IEEE 802.11 distributed coordination function," IEEE J. Sel. Areas Commun., vol. 18, no. 3, pp. 535-547, Mar. 2000.

[4] J. An, X. Guo, and Y. Yang, "Analysis of collision probability in vehicular ad hoc networks," in Proc. 1st ACM SIGEVO Summit Genetic Evol. Comput., 2009, pp. 791-794.

[5] W. Tan, W. Lau, and O. Yue, "Modeling resource sharing for a road-side access point supporting drive-thru internet," in Proc. ACM VANET, 2009, pp. 33-42.

[6] A. Nandan, S. Das, G. Pau, M. Gerla, and M. Y. Sanadidi, "Co-operative downloading in vehicular ad-hoc wireless networks," in Proc. WONS, 2005, pp. 32-41.

[7] J. Zhao, Y. Zhang, and G. Cao, "Data pouring and buffering on the road: A new data dissemination paradigm for vehicular ad hoc networks," IEEE Trans. Veh. Technol., vol. 56, no. 6, pp. 3266-3277, Nov. 2007.

[8] V. Bychkovsky, B. Hull, A. Miu, H. Balakrishnan, and S. Madden, "A measurement study of vehicular Internet access using in situ Wi-Fi networks," in Proc. ACM MobiCom, 2006, pp. 50-61.

[9] D. Hadaller, S. Keshav, T. Brecht, and S. Agarwal, "Vehicular opportunistic communication under the microscope," in Proc. ACM MobiSys, 2007, pp. 206-219.

[10] V. Shrivastava, N. Ahmed, S. Rayanchu, S. Banerjee, S. Keshav, K. Papagiannaki, and A. Mishra, "CENTAUR: Realizing the full potential of centralized WLANs through a hybrid data path," in Proc. ACM MobiCom, 2009, pp. 297-308.

[11] Z. Zeng, Y. Gao, T. Kun, and P. R. Kumar, "CHAIN: Introducing minimum controlled coordination into random access MAC," in Proc. IEEE INFOCOM, 2011, pp. 2669-2677.

[12] M. J. Grace and R. B. Potts, "A theory of the diffusion of traffic platoons," Oper. Res., vol. 12, no. 2, pp. 255-275, Mar./Apr. 1964.

[13] F. Bai and B. Krishnamachari, "Spatio-temporal variations of vehicle traffic in VANETs: Facts and implications," in Proc. ACM VANET, 2009, pp. 43-52.

[14] W. Alasmary and W. Zhuang, "Mobility impact in IEEE 802.11p infrastructureless vehicular networks," Ad Hoc Netw., vol. 10, no. 2, pp. 222-230, Mar. 2012.

[15] W. Heinzelman, "Application-specific protocol architectures for wireless networks," Ph.D. dissertation, Mass. Inst. Technol., Cambridge, MA, 2000.

[16] J. D. Fricker and R. K. Whitford, Fundamentals of Transportation Engineering: A Multimodal Systems Approach. Upper Saddle River, NJ: Prentice-Hall, 2004.

[17] Part 11: Wireless LAN Medium Access Control (MAC) and Physical Layer (PHY) specifications, IEEE Std. 802.11-1999, 1999.

[18] L. X. Cai, X. Shen, L. Cai, J. Mark, and Y. Xiao, "Voice capacity analysis of WLAN with un-balanced traffic," IEEE Trans. Veh. Technol., vol. 55, no. 3, pp. 752-761, May 2006.

[19] X. Ling, "Performance analysis of distributed MAC protocols for wireless networks," Ph.D. dissertation, Univ. Waterloo, Waterloo, ON, Canada, 2007.

[20] X. Ling, Y. Cheng, J. W. Mark, and X. Shen, "A renewal theory based analytical model for the contention access period of IEEE 802.15.4 MAC," IEEE Trans. Wireless Commun., vol. 7, no. 6, pp. 2340-2349, Jun. 2008.

[21] T. van Woensel and N. Vandaele, "Modeling traffic flows with queueing models: A review," Asia-Pac. J. Oper. Res., vol. 24, no. 4, pp. 435-461, 2007.

[22] M. Cheung, F. Hou, V. Wong, and J. Huang, "DORA: Dynamic optimal random access for vehicle-to-roadside communications," IEEE J. Sel. Areas Commun., to be published. 


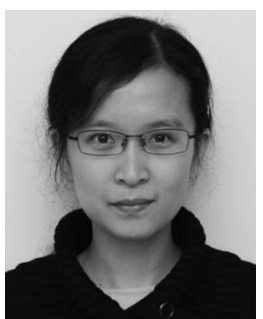

Yanyan Zhuang (S'09) received the B.E. and M.E. degrees in computer science and engineering from Southeast University, Nanjing, China, in 2005 and 2008 , respectively. She is currently working towards the Ph.D. degree with the Department of Computer Science, University of Victoria, Victoria, BC, Canada.

Her research interests include the theory and application of geometrical probability and span the areas of network protocols and system design and analysis in wireless communications and technologies. Her research focus is in wireless sensor networks and vehicular ad hoc networks.

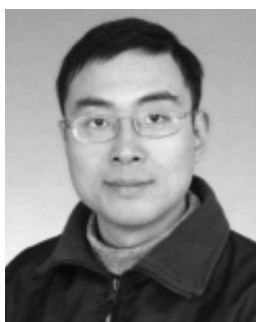

Jianping Pan (S'96-M'98-SM'08) received the B.E. and Ph.D. degrees in computer science from Southeast University, Nanjing, China, and did postdoctoral research with the University of Waterloo, Waterloo, ON, Canada.

He is currently an Associate Professor of computer science with the University of Victoria, Victoria, BC, Canada. He was also with Fujitsu Laboratories and NTT Laboratories. His area of specialization is computer networks and distributed systems. His current research interests include protocols for advanced networking, performance analysis of networked systems, and applied network security.

Dr. Pan received the Institute of Electronics, Information, and Communication Engineers Best Paper Award in 2009, the Telecommunications Advancement Foundation's Telesys Award in 2010, the International Conference on Wireless Communications and Signal Processing 2011 Best Paper Award, and the IEEE Global Communications Conference (Globecom), 2011 Best Paper Award. He has been serving on the Technical Program Committees of major computer communications and networking conferences, including the IEEE International Conference on Computer Communications, the IEEE International Conference on Communications, Globecom, the IEEE Wireless Communications and Networking Conference, and the IEEE Consumer Communications and Networking Conference. He is a Senior Member of the Association for Computing Machinery.

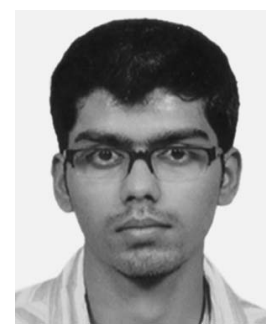

Vidhoon Viswanathan received the B.Tech. degree in information technology from Anna University, Chennai, India, in 2011.

$\mathrm{He}$ is currently a Design Engineer with Texas Instruments Research and Development, Bangalore, India. His research interests include wireless communication, mobile computing, multicore programming, and artificial intelligence.

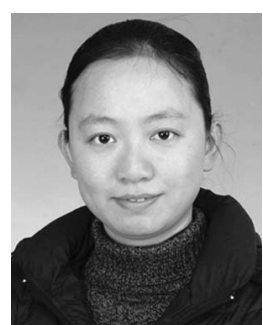

Lin Cai (S'00-M'06-SM'10) received the M.A.Sc. and $\mathrm{Ph} . \mathrm{D}$. degrees in electrical and computer engineering from the University of Waterloo, Waterloo, ON, Canada, in 2002 and 2005, respectively.

Since 2005, she has been an Assistant Professor and then an Associate Professor with the Department of Electrical and Computer Engineering, University of Victoria, Victoria, BC, Canada. Her research interests include wireless communications and networking, with a focus on network protocol and architecture design supporting emerging multimedia traffic over wireless, mobile, ad hoc, and sensor networks.

Dr. Cai received the Natural Sciences and Engineering Research Council Discovery Accelerator Supplement Grant in 2010. She also received the Best Paper Awards at the 2008 IEEE International Conference on Communications and the 2011 IEEE Wireless Communications and Networking Conference. She has served as Associate Editor for the IEEE TRANSACTIONS ON Vehicular TeChNOLOGY, the IEEE TRANSACTIONS ON Wireless COMmUniCATIOnS, the EURASIP Journal on Wireless Communications and Networking, the International Journal of Sensor Networks, and the Journal of Communications and Networks. 